\title{
Intergenic and External Transcribed Spacers of Ribosomal RNA Genes in Lizard-infecting Leishmania: Molecular Structure and Phylogenetic Relationship to Mammal-infecting Leishmania in the Subgenus Leishmania (Leishmania)
}

\author{
Tereza C Orlando, Mary Anne T Rubio*, Nancy R Sturm*, David A Campbell*, \\ Lucile M Floeter-Winter/ ${ }^{+}$
}

\begin{abstract}
Departamento de Parasitologia, Instituto de Ciências Biomédicas, Universidade de São Paulo, Av. Prof. Lineu Prestes 1374, 05508-900 São Paulo, SP, Brasil *Department of Microbiology, Immunology \& Molecular Genetics, University of California, Los Angeles, USA
\end{abstract}

To establish the relationships of the lizard-and mammal-infecting Leishmania, we characterized the intergenic spacer region of ribosomal RNA genes from $\mathrm{L}$. tarentolae and $\mathrm{L}$. hoogstraali. The organization of these regions is similar to those of other eukaryotes. The intergenic spacer region was approximately $4 \mathrm{~kb}$ in $\mathrm{L}$. tarentolae and 5.5 $k b$ in $\mathrm{L}$. hoogstraali. The size difference was due to a greater number of 63-bp repetitive elements in the latter species. This region also contained another element, repeated twice, that had an inverted octanucleotide with the potential to form a stem-loop structure that could be involved in transcription termination or processing events. The ribosomal RNA gene localization showed a distinct pattern with one chromosomal band (2.2 Mb) for L. tarentolae and two (1.5 and $1.3 \mathrm{Mb}$ ) for $\mathrm{L}$. hoogstraali. The study also showed sequence differences in the external transcribed region that could be used to distinguish lizard Leishmania from the mammalian Leishmania. The intergenic spacer region structure features found among Leishmania species indicated that lizard and mammalian Leishmania are closely related and support the inclusion of lizard-infecting species into the subgenus Sauroleishmania proposed by Saf'janova in 1982.

Key words: lizard leishmania - RNA polymerase I - rRNA processing - repetitive DNA - rRNA spacer evolution

Leishmania tarentolae and L. hoogstraali are bloodstream kinetoplastids of lizards. Their geographical distribution is restricted to the Old World. Lizard-infecting Leishmania are closely related to mammalian-infecting Leishmania, but their exact taxonomic position and phylogenetic relationship remains uncertain. Recently, biochemical and biological data have been used in comparative studies of lizard and mammalian Leishmania species (Simpson \& Holz 1988, Briones et al. 1992, Fu \& Kolesnikov 1994, Croan \& Ellis 1996, Previato et al. 1997, Noyes et al. 1997, Croan et al. 1997, Shaw 1997, Noyes et al. 1998) indicating a close relationship.

Lizard-infecting Leishmania play an important role in epidemiological studies of leishmaniasis. Amastigote forms of L. adleri (another lizard Leishmania species) were detected in volunteers at the site of inoculation five

This work was supported by Fapesp (Fundação de Amparo à Pesquisa do Estado de São Paulo), CNPq and NIH (Grant no. AI34536). TCO was supported by a fellowship from Fapesp and MATR was supported by a National Science Foundation Graduate Research Fellowship.

Note: Nucleotide sequence data reported here are available in the GenBank database under the accession numbers AY026525 and AF345631-33.

+Corresponding author. Fax: +55-11-3091.7329. E-mail: lmfwinte@icb.usp.br

Received 13 September 2001

Accepted 6 February 2002 days after infection with L. adleri promastigotes. Serological studies also showed common antigens among $L$. adleri and L. hoogstraali and some mammalian Leishmania species. This observation has an implication in the kala-azar epidemiology since false positives could be generated due to naturally-transmitted L. adleri (see revision in Wilson \& Southgate 1979, Telford 1995). In addition, amastigote forms have been detected in phagocytic cells of naturally and experimentally infected lizards (Dollahon \& Janovy 1974, Elwasila 1988).

Earlier studies using the small subunit ribosomal RNA (rRNA) molecule helped to establish the lizard Leishmania proximity to mammalian Leishmania (Briones et al. 1992) relative to other trypanosomatid genera. However, the small subunit sequences are not suitable for resolution of species within the same genera. On the other hand, the rRNA gene intergenic spacer region (IGS) and the external transcribed spacer (ETS) are under less selective pressure and thus provide a means to distinguish evolutionary relationships among more closely-related species. The IGS/ETS region of trypanosomatids have been widely studied (White et al. 1986, Grondal et al. 1990, Zomerdijk et al. 1991, Rudenko et al. 1991, Dietrich et al. 1993, Martinez-Calvillo \& Hernandez 1994, Tyler-Cross et al. 1995, Uliana et al. 1996, Gay et al. 1996, Yan et al. 1999, Downey \& Donelson 1999, Schnare et al. 2000) and shows a general organization similar to the majority of eukaryotes.

In the present work, the molecular structure of the IGS region from two lizard-infecting Leishmania species is described and compared to the corresponding sequences 
from the mammal-infecting Leishmania. These data refine the phylogenetic positioning of the lizard Leishmania and support their status as a subgenus of Leishmania.

\section{MATERIALS AND METHODS}

Organisms - Promastigotes of L. tarentolae (ATCC 30267) and L. hoogstraali (RHEM/SD/1963/NG26) from the Trypanosomatid Bank at Universidade de São Paulo were cultivated at $25^{\circ} \mathrm{C}$ in LIT medium (Camargo 1964).

DNA and RNA extractions, isolation of the IGS/ETS region and sequencing - Genomic DNA from Leishmania species was purified as previously described (Uliana et al. 1991). Total RNA was extracted as described (Chomczynski \& Sacchi 1987).

A genomic ApaI-BglII fragment encompassing $\sim 3 \mathrm{~kb}$ of the IGS/ETS region of L. tarentolae was isolated from a cosmid library (Fig. 1C, clone pAB 2.8). Clone pLt1.3 (Fig. 1C) was obtained by the isolation of an AluI fragment from pAB 2.8. For L. hoogstraali, a 9-kb BglII fragment was isolated from a partial genomic library, constructed using inserts of 7 to $10 \mathrm{kB}$ produced by a complete digestion of genomic DNA with $B g l$ I. This fragment contains $\sim 2 \mathrm{~kb}$ from the large subunit rRNAs subunits and the whole IGS/ETS region. Fig. 1B shows clones $\mathrm{pLh}$ 6500, pLh 3400 and pLh 8A, containing small fragments, used in this work. These clones were mapped with several restriction enzymes, subcloned into pUC 18/19 series (Fig. 1) and sequenced by the dideoxynucleotide method (Sanger et al. 1977). The sequences were determined by a combination of manual and automated sequencing using an ABI 377 or 310 sequencer (Perkin Elmer).
Chromosomal analysis - Chromosome bands were separated by pulsed-field gel electrophoresis (Schwartz \& Cantor 1984) in 1.2\% agarose gels and $0.5 x$ TBE buffer. The running conditions were: three phases pulses of 180 , 240 and $300 \mathrm{~s}$ at $6 \mathrm{~V} / \mathrm{cm}$ for $18 \mathrm{~h}$ each pulse at $10^{\circ} \mathrm{C}$. Blots were probed with the AccI fragment (Fig. 1C) and with the small subunit rRNA from L. tarentolae.

Sequence and phylogenetic analysis - The sequences were aligned with CLUSTALW 1.6 (Thompson et al. 1994) and the matrix obtained contained 1119 characters and 6 taxa. Crithidia fasciculata was used as the outgroup.

Phylogenetic trees were constructed using PAUP version 4.03b (Swofford 1999) for distance and parsimony methods. Maximum likelihood trees were obtained with BASEML program from the PAML 2.0 package (Yang 1999) using the K80 model of DNA substitution with gamma distribution $(\alpha=0.85)$. Bootstrap values were calculated for 100 replicates.

\section{RESULTS}

IGS/ETS sequence comparison - Based in a Northern blot experiment the approximate size of the ETS region of L. hoogstraali and L. tarentolae was determined (data not shown) and used to positioned the data to align and compare this region with the corresponding sequences of L. (L.) amazonensis, L. (L.) chagasi, L. (L.) donovani and $C$. fasciculata (Fig. 2, Table I). The comparison showed blocks of sequence similarity among the Leishmania species and Crithidia. Motifs were identified corresponding to those described previously in $L$. (L.) amazonensis (Uliana et al. 1996) and two regions (Fig. 2) of absolute identity to the proposed base-pairing sites of

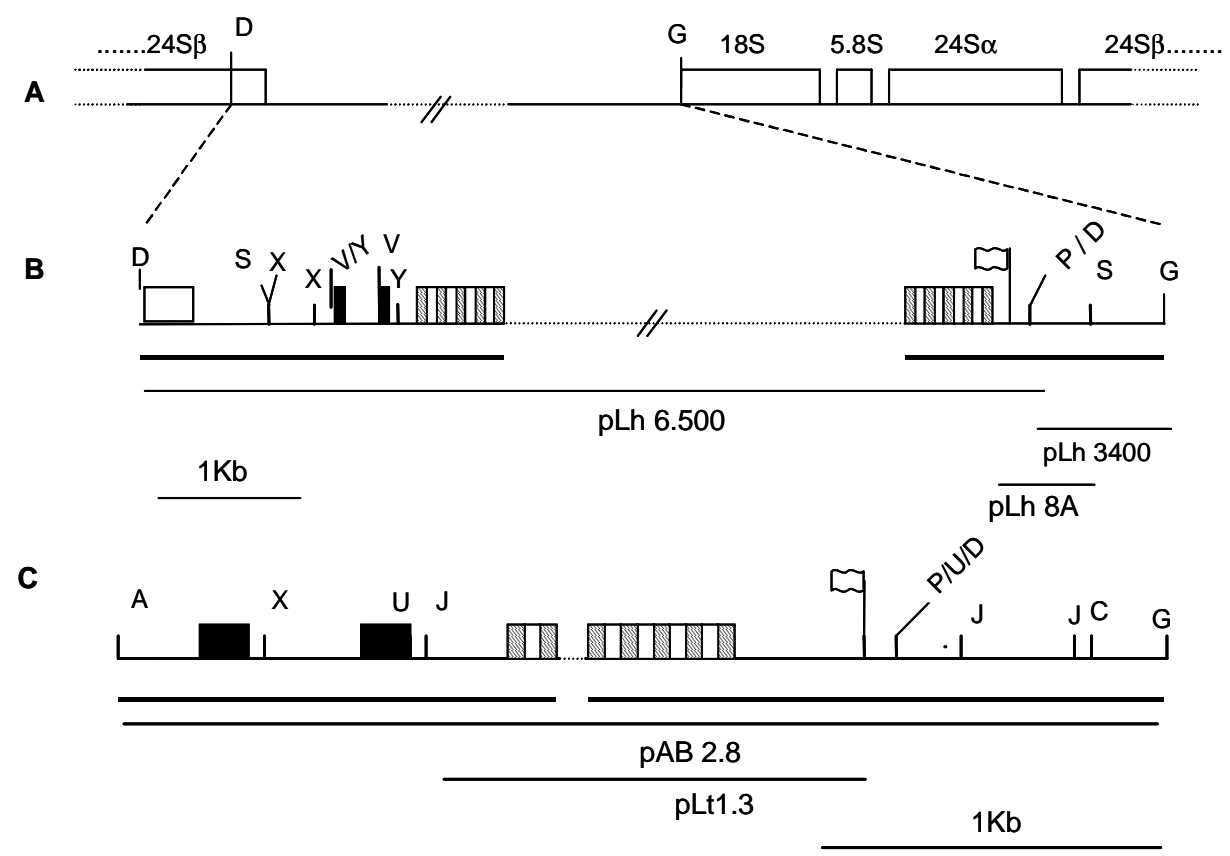

Fig. 1: scheme of the general ribosomal RNA gene arrangement (A) with the respective intergenic spacer/external transcribed spacer region of Leishmania hoogstraali (B) and L. tarentolae (C). The blank box in B represents the 3' end of the rRNA gene 24S $\beta$. A, ApaI; B, BamHI; C, HincII; D, HindIII; G, BglII; J, AccI; P, PstI; S, SmaI; U, AluI; V, PvuII; X, XhoI; Y, DdeI. The 63 bp repetitive elements are represented in stippled and white rectangles, the flag maps the estimated transcription start point, the black rectangles indicate the element containing the inverted octanucleotides. The thick line corresponds to the sequenced region. 
the U3 small nucleolar RNA (snoRNA) of C. fasciculata (Schnare et al. 2000) and L. tarentolae (Shi et al. 1994). A HindIII site in the estimated ETS region of L. tarentolae and L. hoogstraali (Fig. 1) could be used to discriminate lizard isolates from the mammalian isolates.

L. tarentolae and L. hoogstraali IGS/ETS structure The IGS/ETS structure was determined by sequence analysis of the corresponding genomic segments from each species (Fig. 1): $4 \mathrm{~kb}$ for L. tarentolae and $5.5 \mathrm{~kb}$ for L. hoogstraali.

The relevant IGS/ETS fragments were digested with restriction enzymes that had 4-bp recognition sites. The patterns obtained for both species with DdeI revealed the presence of repetitive element in both regions. A nonstoichiometric quantity of a 70-bp fragment accumulated with partial-restriction kinetics, and the sum of all frag- ments generated by complete digestion was less than the size of the original fragment (data not shown).

Determining the nucleotide sequence of the IGS fragments allowed us to fully characterize the repetitive elements. For both species, the first 63-bp repeat is located $\sim 200$ bp upstream of the estimated transcription start point (Fig. 1B, C, hatched rectangles). By sequence analysis we detected approximately 12 repeats for L. tarentolae and 40 for L. hoogstraali.

These repetitive elements showed approximately $90 \%$ sequence identity between lizard Leishmania species (Table I, upper section). In contrast, comparison with the same repetitive elements in the IGS of mammalian Leishmania showed a very low degree of similarity (Table I, upper section). Fig. 3 shows a sequence comparison of the repetitive elements found in each species.

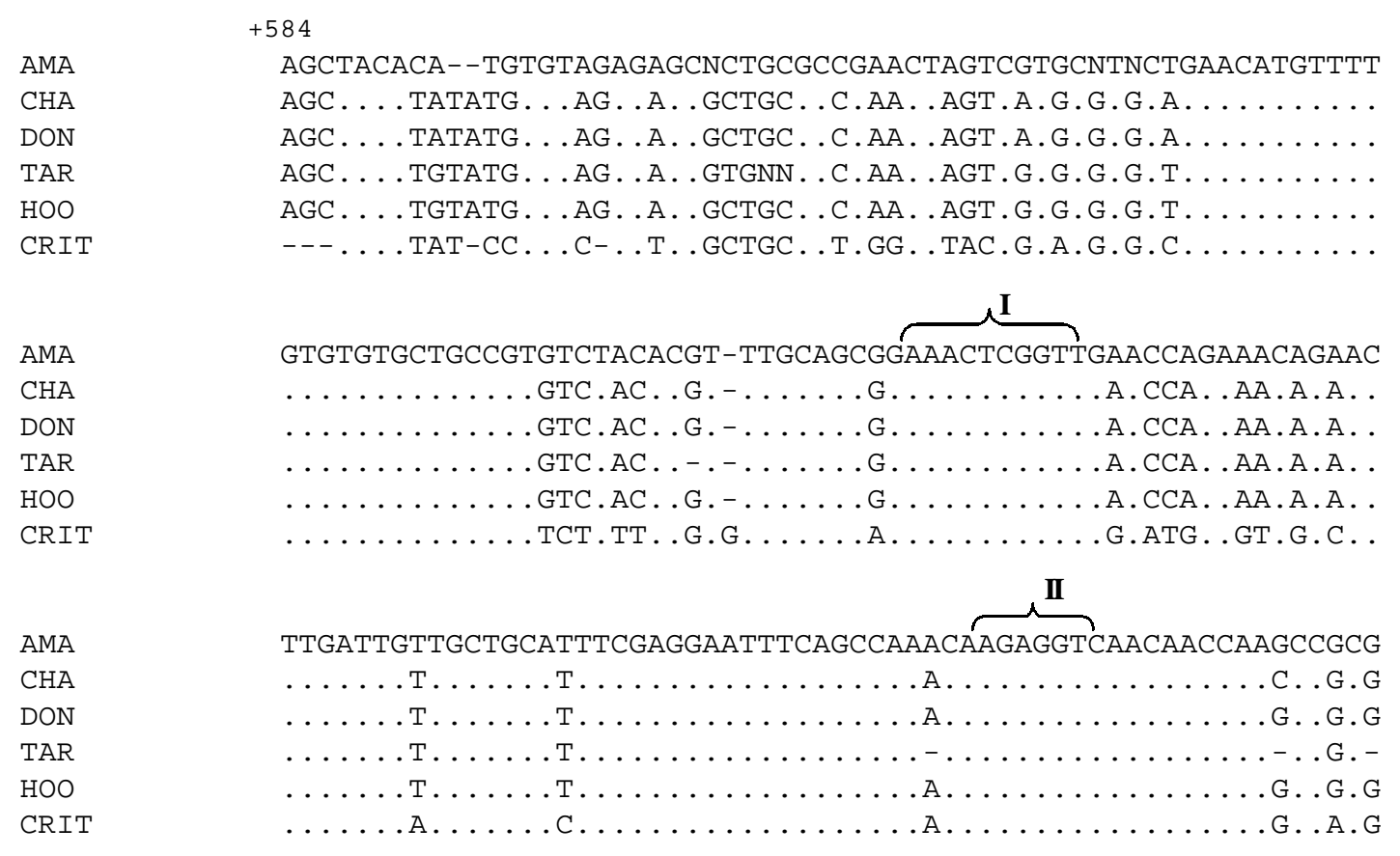

Fig. 2: sequence comparison of a segment of the external transcribed spacer. AMA corresponds to Leishmania (L.) amazonensis, CHA corresponds to $L$. $(L$.) chagasi, DON correspondes to $L$. $(L$.) donovani, TAR corresponds to $L$. tarentolae, HOO corresponds to $L$. hoogstraali and CRIT corresponds to Crithidia fasciculata. GeneBank accession numbers for $L$. (L.) amazonensis, $L$. (L.) chagasi, L. (L.) donovani and C. fasciculata are U21687, U42465, L38572 and Y00055, respectively. Regions I and II correspond to the proposed site of base pairing with the U3 snoRNA in C. fasciculata (Schnare et al. 2000) and L. tarentolae (Shi et al. 1994). Numbering is in relation to the transcription start point of L. amazonensis (Uliana et al. 1996). Dots (.) represent nucleotide identity, dashes (-) represent gaps introduced to improve the alignment obtained with Clustalw (1.60) program.

TABLE

Percentage of similarity among the six external transcribed spacer sequences (lower part) and in the 63bp repetitive elements (upper part)

\begin{tabular}{lccccc}
\hline & L. tarentolae & L. hoogstraali & L. (L.) amazonensis & L. (L.) chagasi & L. (L.) donovani \\
\hline Leishmania tarentolae & - & 90 & 36 & 34 & 36 \\
L. hoogstraali & 93 & - & 35 & 34 & 36 \\
L. (L.) amazonensis & 85 & 87 & - & 68 & 68 \\
L. (L.) chagasi & 85 & 86 & 88 & - & 96 \\
L. L.) donovani & 86 & 88 & 90 & 57 & - \\
Crithidia fasciculata & 60 & 62 & 59 & 58 & 59 \\
\hline
\end{tabular}




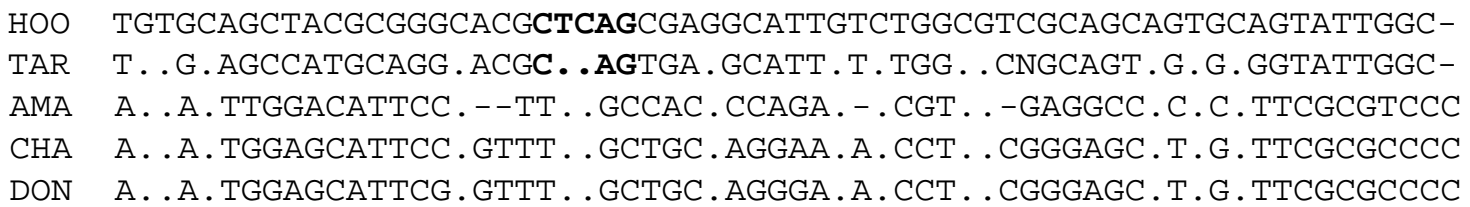

Fig. 3: repetitive elements found in the intergenic spacer region of the rRNA genes of Leishmania species. Species identification as in Fig. 2. Bold type is the DdeI site. Dots (.) represent nucleotide identity, dashes (-) represent gaps introduced to improve the alignment obtained with Clustalw (1.60) program.

The results of the probed pulsed-field gel electrophoresis blots (Fig. 4) indicated that the repetitive elements co-localized to the same chromosomal bands as the rRNA of both species and were not dispersed throughout the genome. The pulse-field pattern also revealed that the rRNA gene maps to chromosomal bands of different size (Fig. 4) in $L$. tarentolae $(2.2 \mathrm{Mb})$ and $L$. hoogstraali (1.5 and $1.3 \mathrm{Mb}$ ). Hybridization experiments using high stringency conditions indicated the presence of these elements in L. adleri and L. gymnodactyli (other lizard Leishmania species). The 63-bp repetitive elements from lizard Leishmania did not hybridize with $L$. ( $L$.) amazonensis nor $L$. (L.) mexicana chromosomal bands (data not shown).

Upstream from the 63-bp repetitive elements, an inverted octanucleotide motif repeated twice was found in both species (Fig. 1B, C, black rectangles). A similar sequence was described in the same IGS position in $L$. $(L$.) infantum (Requeña et al. 1997) and recently, it was also found in the IGS region of $C$. fasciculata (Schnare et al. 2000). A comparison among these sequences showed that the repeats in C. fasciculata and Leishmania species are not as conserved as among the Leishmania species repeats (Fig. 5A). The potential to form a stem-loop struc- ture as identified in the $L$. (L.) infantum sequence was also found for the two blocks of $L$. tarentolae, $L$. hoogstraali and C. fasciculata (Fig. 5B).

Phylogeny - Trees derived from distance, parsimony and maximum likelihood (Fig. 6) methods exhibited identical topologies and showed that the Leishmania of lizards studied are very close to each other. The lizard Leishmania form a sister group to the mammalian Leishmania of the subgenus L. (Leishmania) analyzed with $C$. fasciculata used as the outgroup. The bootstrap values obtained for each method (Fig. 6, nodes) support the hypothesis that lizard Leishmania and mammalian Leishmania species of the subgenus L. (Leishmania) are sister groups.

\section{DISCUSSION}

In this study we have determined the sequence of the IGS/ETS upstream from the small subunit rRNA of two lizard-infecting Leishmania species for comparison with their mammal-infecting relatives. The size of the IGS region determined for both species is approximately the same as the size found for $L$. (L.) infantum ( $5 \mathrm{~kb}$, Requeña et al. 1997 ) and $L$. (L.) donovani (4 kb, Lodes et al. 1995), indicating that the size of this region is conserved between
A

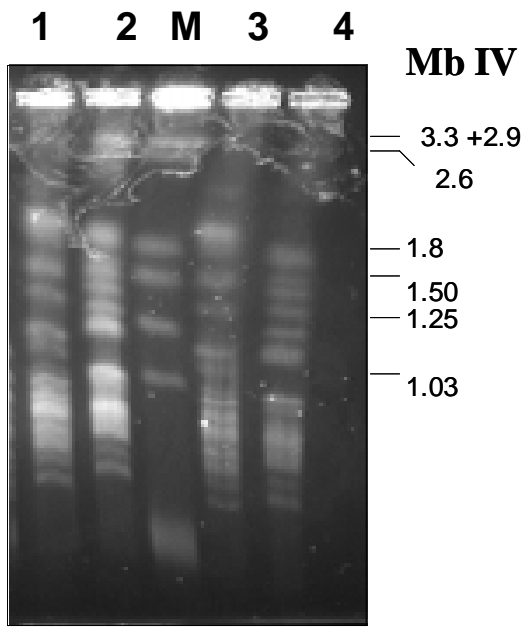

B

I

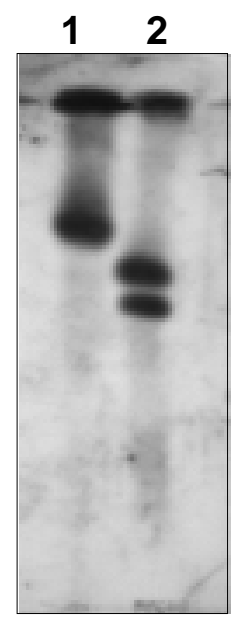

II

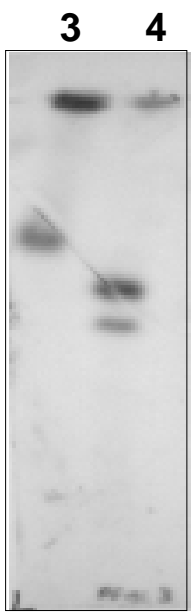

Fig. 4: chromosomal pattern of Leishmania tarentolae (1 and 3) and L. hoogstraali (2 and 4) rRNA gene localization. A: chromosomal bands after ethidium bromide staining. M corresponds to Megabase IV (Invitrogen); B: autoradiogram of the chromosome blot in panel A. In panel I the fragment AccI/AccI from L. tarentolae (Fig. 1A) was used as the probe and in panel II the $18 \mathrm{~S}$ subunit DNA was used as the probe. Washing conditions: $2 \mathrm{x}$ SSC $/ 0.1 \%$ SDS at $45^{\circ} \mathrm{C}$. Exposure time: 5 days at $-20^{\circ} \mathrm{C}$ for panel $\mathrm{I}$ and 4 days at $-20{ }^{\circ} \mathrm{C}$ for panel II. 
A

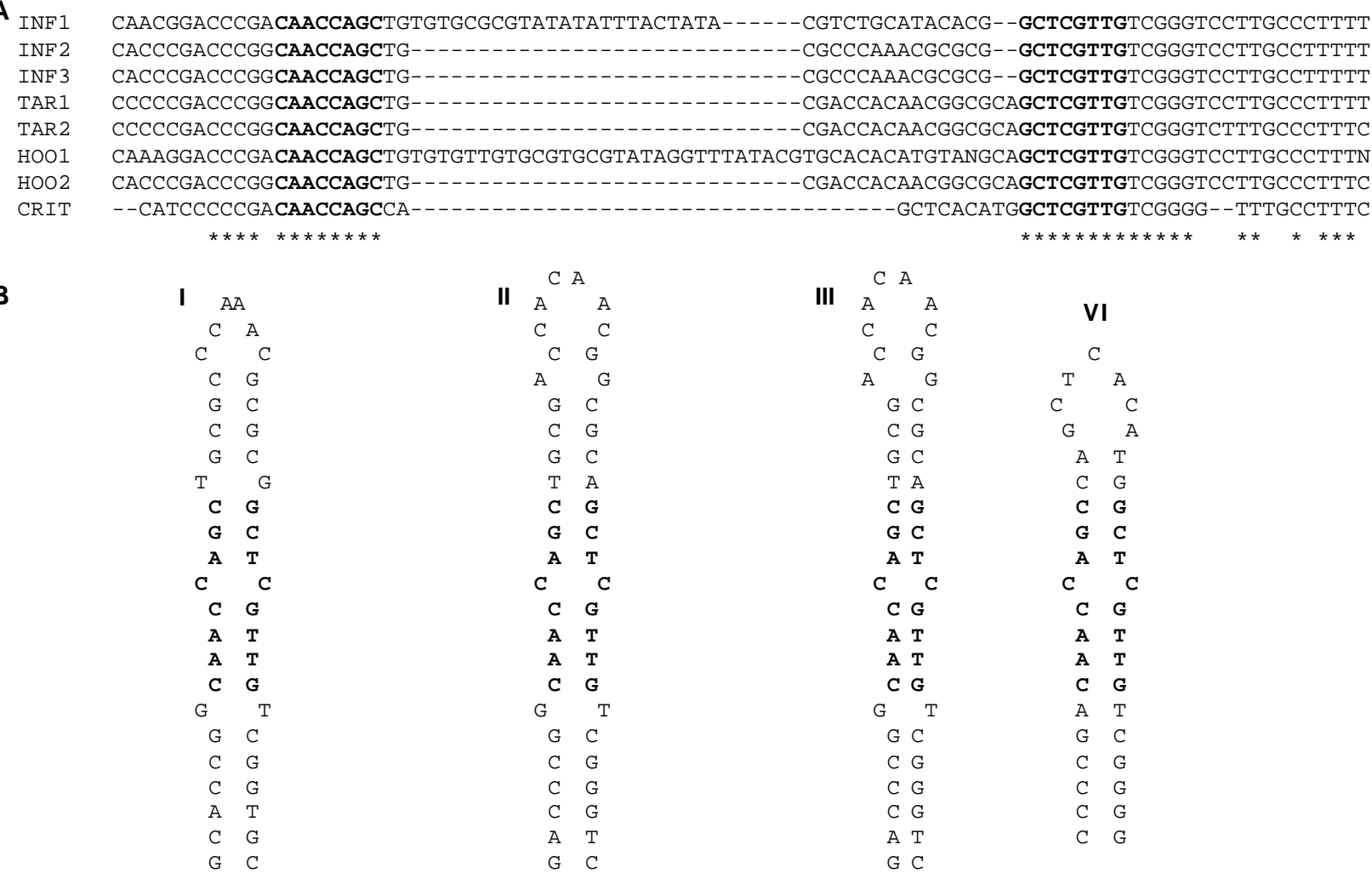

Fig. 5: analysis of the three repetitive elements found downstream from the Leishmania (L.) infantum large subunit rRNA (INF 1, 2 and 3), L. tarentolae (TAR 4 and 5), L. hoogstraali (HOO 6 and 7) and Crithidia fasciculata (CRIT). A: alignment of the elements. Bold letters correspond to the inverted octanucleotides. Asterisks (*) below the alignment indicate base identity in each position among the eight sequences; dashes (-) represent gaps introduced to maximize the alignment; B: sequences shown in A can form similar stem loop structures. I. L. (L.) infantum (Requeña et al. 1997); II. L. tarentolae. III. L. hoogstraali. IV. C. fasciculata (from R3 sequence described in Schnare et al. 2000).

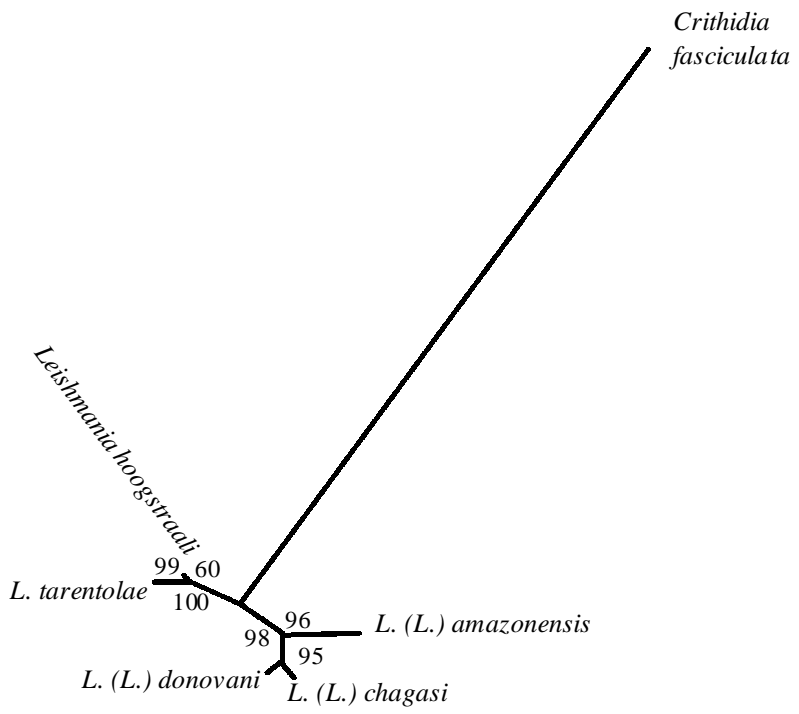

0.1

Fig. 6: topology obtained with the maximum likelihood method for the external transcribed spacer sequence alignment. Distance method (performed with the neighbor-joining algorithm) and parsimony method gave similar topologies, although the bootstrap values obtained differed: numbers at the left of each node represent maximum likelihood values, numbers at the right represent neighborjoining values and numbers under each node represent parsimony values. The bar indicates distance for maximum likelihood. lizard and mammalian Leishmania species; the size observed in other eukaryotes is variable. The differences in length can be attributed to a variable number of $63-b p$ repetitive-element found in each lizard species.

The estimated ETS region of each species ( 1000 bp) was shown to be very similar in size to $L$. $(L$.) amazonensis (1050 bp, Uliana et al. 1996), L. (L.) chagasi (1060 bp, Gay et al. 1996), L. (L.) donovani (1020 bp, Yan et al. 1999), C. fasciculata (1050 bp, Grondal et al. 1990) and T. congolense (961 bp, Downey \& Donelson 1999). For T. cruzi and T. brucei the ETS size is significantly larger [1550 bp, Dietrich et al. (1993) and $1200 \mathrm{bp}$, White et al. (1986), respectively].

The comparison of ETS regions showed conserved motifs between lizard and mammalian Leishmania species and $C$. fasciculata (data not shown), including the proposed sites of base-pairing with the U3 snoRNA of $C$. fasciculata (Schnare et al. 2000) and L. tarentolae (Shi et al. 1994) (Fig. 2). The presence of similar motifs suggests a common processing mechanism of the pre-rRNA molecule.

As in many other eukaryotes, conserved, repeated elements upstream of the predicted transcription start point were detected in both species. However, when compared to mammalian Leishmania species, the degree of similarity dropped three fold (Table). The repeat number varied among the Leishmania species: two populations of 35 and 70 copies in $L$. (L.) amazonensis (Uliana et al. 1996) 
and 39 copies in $L$. ( $L$.) donovani (Yan et al. 1999), while for lizard Leishmania approximately 12 copies of the 63bp repeat in the $L$. tarentolae IGS and approximately 40 copies in L. hoogstraali were found. The size of the repeated elements was conserved in Leishmania ( 60 bp), while 28 copies of a 19-bp element were found located upstream of the transcription start point in $C$. fasciculata (Schnare et al. 2000), indicating that the repeat size is not necessarily conserved in the kinetoplastids. In $L$. $(L$.) chagasi these elements showed a modest enhancer activity in a transient transfection assay (Gay et al. 1996).

The chromosomal analysis (Fig. 4) showed that the rRNA genes and the 63-bp repetitive elements mapped to the same chromosomal bands for both species. However, the detection of two chromosomal bands of different size in L. hoogstraali (1.3 and $1.5 \mathrm{Mb}$ ) does not discriminate whether they reside on homologous or different chromosomes. A similar pattern of two chromosomal bands for the rRNA genes was also identified for some Viannia subgenus species (Inga et al. 1998), as well as in $L$. (L.) donovani (Yan et al. 1999).

The analysis of an element located downstream of the large subunit 2 (24S $\beta$ ) of $L$. tarentolae, $L$. hoogstraali, $L$. (L.) infantum (Requeña et al. 1997) and C. fasciculata (Schnare et al. 2000) showed high degrees of similarity, mainly in the inverted octanucleotides (Fig. 5A, bold) and in the nucleotides that can form potential stem-loop structures (Fig. 5B).

This conservation indicates a possible biological role to this element, probably in the transcription termination of pre-rRNA or in subsequent processing events. The sequence of the element is conserved among Leishmania species, demonstrating the close relationship between lizard and mammalian Leishmania. An intriguing aspect of these motifs is that they have inverted octanucleotides identical to blocks found in 1 phage and Escherichia coli (Thaler \& Stahl 1988), where they define hot spots for recombination. Thus, the repeated sequences could be involved in recombination events, as evidenced by the correlation of the repetitive sequences to the heterogeneity in the copy number of the rRNA genes.

The alignment of ETS sequences showed a high degree of sequence similarity between lizard Leishmania and mammal-infecting Leishmania (Table, lower section). The analysis of the data using diverse phylogenetic approaches resulted in the generation of identical trees showing that the lizard Leishmania are very closely related to $L$. (L.) donovani. These results are in agreement with biogeographic data, since both lizard Leishmania and $L$. (L.) donovani are found only in the Old World (Shaw 1994). Similar phylogenetic relationships for these organisms were found using the DNA polymerase I genes and RNA polymerase II genes of different Leishmania species (Croan et al. 1997). Furthermore, functional results involving the exact transcription starting point, the ETS and the promoter region of the rRNA gene of lizard Leishmania showed some degree of heterologous recognition of these regions between lizards and mammalian-infecting species (Orlando et al., manuscript in preparation), corroborating their close relationship also in a functional level, as this recognition is thought to be highly speciesspecific (Grummt et al. 1982, Sommerville 1984).
Both the molecular data presented and the inferred phylogenetic relationships are in agreement with the inclusion of the lizard Leishmania in the subgenus Sauroleishmania, as proposed by Saf'janova, using biological criteria (Saf'janova 1982).

\section{ACKNOWLEDGEMENTS}

To Jeffrey J Shaw for critical reading and comments on the manuscript and Sergio Pereira for help with the phylogeny.

\section{REFERENCES}

Briones MRS, Nelson K, Beverley SM, Affonso MHT, Camargo EP, Floeter-Winter LM 1992. Leishmania tarentolae: taxonomic relatedness inferred from phylogenetic analysis of small subunit ribosomal RNA gene. Mol Biochem Parasitol 53: 121-128.

Camargo EP 1964. Growth and differentiation of Trypanosoma cruzi. I. Origin of metacyclic trypanosomes in liquid media. Rev Inst Med Trop São Paulo 6: 93-100.

Chomczynski P, Sacchi N 1987. Single-step method of RNA isolation by acid guanidinium thiocyanate-phenol-chloroform extraction. Anal Biochem 162: 156-159.

Croan DA, Ellis J 1996. Phylogenetic relationship between Leishmania, Viannia and Sauroleishmania inferred from comparison of a variable domain within the RNA polymerase II largest subunit gene. Mol Biochem Parasitol 79: 97-102.

Croan DA, Morrison DA, Ellis J 1997. Evolution of the genus Leishmania revealed by comparison of DNA and RNA polymerase gene sequences. Mol Biochem Parasitol 89: 149-159.

Dietrich P, Soares MBM, Affonso MHT, Floeter-Winter LM 1993. Trypanosoma cruzi ribosomal gene: analysis of promoter and upstream intergenic spacer sequences. Gene 125: 103-107.

Dollahon NR, Janovy Jr J 1974. Experimental infection of New World lizards with Old World lizard Leishmania species. Exp Parasitol 36: 253-260.

Downey N, Donelson JE 1999. Search for promoters for GARP and rRNA genes of Trypanosoma congolense. Mol Biochem Parasitol 104: 25-38.

Elwasila M 1988. Leishmania tarentolae Wenyon, 1921 from the gecko Tarentola annularis in the Sudan. Parasitol Res 74: 591-592.

Fu O, Kolesnikov AA 1994. Leishmania gymnodactyli and Leishmania infantum minicircles contain the same guide RNA genes as do minicircles of Leishmania tarentolae. Mol Biochem Parasitol 67: 171-174.

Gay LS, Wilson ME, Donelson JE 1996. The promoter for ribosomal RNA genes of Leishmania chagasi. Mol Biochem Parasitol 77: 115-252.

Grondal EJM, Evers R, Cornelissen AWCA 1990. Identification and sequence analysis of the ribosomal DNA promoter region of Crithidia fasciculata. Nucleic Acids Res 18: 13331338.

Grummt I, Roth E, Paule MR 1982. Ribosomal RNA transcription in vitro is species specific. Nature 296: 173-174.

Inga R, De Doncker S, Gomez J, Lopez M, Garcia R, Le Ray D, Arevalo J, Dujardin JC 1998. Relation between variation in copy number of ribosomal RNA encoding genes and size of harbouring chromosomes in Leishmania of subgenus Viannia. Mol Biochem Parasitol 92: 219-228.

Lodes MJ, Merlin G, Devos T, Ghosh A, Madhubala R, Myler PJ, Stuart K 1995. Increased expression of LD1 genes transcribed by RNA polymerase I in Leishmania donovani as a result of duplication into the rRNA gene locus. Mol Cell 
Biol 15: 6845-6853.

Martinez-Calvillo S, Hernandez R 1994. Trypanosoma cruzi ribosomal DNA: mapping of a putative distal promoter. Gene 142: 243-247.

Noyes HA, Arana BA, Chance ML, Maingon R 1997. The Leishmania hertigi Kinetoplastida; Trypanosomatidae complex and the lizard Leishmania: their classification and evidence for a neotropical origin of the Leishmania-Endotrypanum clade. J Eukaryot Microbiol 44: 511-517.

Noyes HA, Chance ML, Croan DG, Ellis JT 1998. Leishmania (Sauroleishmania): a comment on classification. Parasitol Today 14: 167.

Previato JO, Jones C, Wait R, Routier F, Saraiva E, MendonçaPreviato L 1997. Leishmania adleri, a lizard parasite, expresses structurally similar glycoinositolphospholipids to mammalian Leishmania. Glycobiol 7: 687-695.

Requeña MJ, Soto M, Quijada L, Carrillo G, Alonso C 1997. A region containing repeated elements is associated with transcriptional termination of Leishmania infantum ribosomal RNA genes. Mol Biochem Parasitol 84: 101-110.

Rudenko G, Chung H-MM, Pham VP, Van Der Ploeg LHT 1991. RNA polymerase I can mediate expression of CAT and neo protein-coding genes in Trypanosoma brucei. EMBO J 10: 3387-3397.

Saf'janova VM 1982. [The problem of taxonomy with Leishmania]. Ser Protozool Sov Acad Sci Leningr 7: 5-109.

Sanger F, Nicklen S, Coulson AR 1977. DNA sequencing with chain terminating inhibitors. Proc Natl Acad Sci USA 74: 5463-5467.

Schnare MN, Collings JC, Spencer DF, Gray MW 2000. The 28S-18S rDNA intergenic spacer from Crithidia fasciculata: repeated sequences, length heterogeneity, putative processing sites and potential interactions between U3 small nucleolar RNA and the ribosomal RNA precursor. Nucleic Acids Res 28: 3452-3461.

Schwartz DC, Cantor CR 1984. Separation of yeast chromosome-sized DNAs by pulsed field gel eletrophoresis. Cell 37: 67-75.

Shaw JJ 1997. Ecological and evolutionary pressures on leishmanial parasites. Braz J Genet 20: 123-128.

Shaw JJ 1994. Taxonomy of the genus Leishmania: present and future trends and their implications. Mem Inst Oswaldo Cruz 89: 471-478.

Shi X, Chen DT, Suyama Y 1994. A nuclear tRNA gene cluster in the protozoa Leishmania tarentolae and differential distribution of nuclear-encoded tRNAs between the cytosol and mitochondria. Mol Biochem Parasitol 65: 23-37.
Simpson L, Holz G 1988. The status of Leishmania tarentolae/ Trypanosoma platydactyli. Parasitol Today 4: 115-118.

Sommerville J 1984. RNA polymerase I promoters and transcription factors. Nature 310: 189-190.

Swofford DL 1999. PAUP* Phylogenetic Analysis Using Parsimony* and other methods. Version 4. Sinauer Associates, Sunderland, Massachusets.

Telford Jr SR 1995. The Kinetoplastid hemoflagellates of reptiles. In JP Kreier, Parasitic Protozoa, Vol. 10, 2nd ed., Academic Press, New York, p. 161-223.

Thaler DS, Stahl FW 1988. DNA double-chain breaks in recombination of phage and of yeast. Ann Rev Genet 22: 169197.

Thompson JD, Higgins DG, Gibson TJ 1994. CLUSTALW: improving the sensitivity of progressive multiple sequence alignments through sequence weighting, position specific gap penalties and weight matrix choice. Nucleic Acids Res 22: 4673-4680.

Tyler-Cross RE, Harris-Kerr C, Floeter-Winter LM, Buck GA 1995. Transient expression mediated by the Trypanosoma cruzi rRNA promoter. Mol Biochem Parasitol 72: 23-31.

Uliana SRB, Affonso MHT, Camargo EP, Floeter-Winter LM 1991. Leishmania: genus identification based on a specific sequence of the 18S ribosomal RNA sequence. Exp Parasitol 72: $157-163$.

Uliana SRB, Fischer W, Stempliuk VA, Floeter-Winter LM 1996. Structural and functional characterization of Leishmania amazonensis ribosomal RNA promoter. Mol Biochem Parasitol 76: 245-255.

White TC, Rudenko G, Borst P 1986. Three small RNAs within the $10 \mathrm{kB}$ trypanosome rRNA transcription unit are analogous to Domain VII of other eukaryotic 28S rRNA. Nucleic Acids Res 14: 9472-9489.

Wilson VCLC, Southgate BA 1979. Lizard Leishmania. In WHR Lumsden, DA Evans (eds), Biology of the Kinetoplastida, Vol. 2, Academic Press, London and New York, p. 241-268.

Yan S, Lodes MJ, Fox M, Myler PJ, Stuart K 1999. Characterization of the Leishmania donovani ribosomal RNA promoter. Mol Biochem Parasitol 103: 197-210.

Yang Z 1999. Phylogenetic analysis by maximum likelihood PAML. Version 2.0. University of College London, program distributed by the author.

Zomerdijk JCBM, Kieft R, Shiels PG, Borst P 1991. Alphaamanitin-resitant transcription units in trypanosomes: a comparison of promoter sequences for a VSG gene expression site and for the ribosomal RNA genes. Nucleic Acids Res 19: 5153-5158. 
